\title{
A new approach for automated location of active segments in intracardiac electrograms
}

\author{
M. P. Nguyen ${ }^{1}$, C. Schilling ${ }^{1}$ and O. Dössel ${ }^{1}$ \\ ${ }^{1}$ Institute of Biomedical Engineering, Universität Karlsruhe (TH), Karlsruhe, Germany
}

\begin{abstract}
Areas in atrium tissue with complex fractionated atrial electrograms (CFAEs) are among others responsible for the maintenance of atrial fibrillation (AFib). Those areas are ideal target sites for ablation to eliminate AFib and restore normal rhythm. An automated identification of CFAEs with signal processing algorithms is essential to develop an objective strategy for AFib ablation. This paper introduces a new approach to locate signal complexes corresponding to electrophysiological activity. The idea behind this algorithm is based on the idea of Pan-Tompkins' QRS-detection algorithm. However in this approach, the extracted signal feature is the signal energy and therefore the algorithm takes into account not only information of the frequency but also of the amplitude. With adaptive thresholding the algorithm is capable to manage changes in the signal dynamics. The results were validated by experts and the algorithm shows a robust performance.
\end{abstract}

Keywords - Atrial Fibrillation, Catheter Ablation, CFAEs, Signal Processing, Segmentation

\section{INTRODUCTION}

Atrial fibrillation (AFib) is the most common cardiac arrhythmia and is responsible for substantial morbidity and mortality in general population. Complex approaches have been developed for the treatment of AFib where radiofrequency ablation is an established curative method.

AFib is characterized by rapid and uncoordinated atrial activities resulting in an irregular ventricular response. Allessie et al. reported that there are two major underlying mechanisms of AFib, i.e. random wavelet re-entry and leading circle re-entry [1]. It is shown by Haissaguerre et al. that ectopic impulses originating in the pulmonary veins often initiate AFib [2]. Furthermore, Nademanee et al. suggested that areas in atrium tissue with complex fractionated atrial electrograms (CFAEs) are responsible for the maintenance of AFib [3]. Thus, in order to achieve conversion of persistent AFib to normal rhythm, areas with CFAEs are to be ablated along with pulmonary veins isolation and linear lesions [4].

Electrophysiologic studies on patients provide the physicians with information to design ablation strategies for the individual. In order to locate areas to be ablated, CFAEs or signal complexes, which are related to electrical activity of electropathological substrate, must be identified. Till now, this procedure is highly dependent on the experience and subjective assessment of the electrophysiologist. For that reason, effort in signal processing has been made to describe the characteristics of electrical activities in atrial electrograms during AFib in either time or frequency domain [5] [6].

There are two commercial mapping systems, CARTO (Biosense-Webster) and EnSite NavX system (St. Jude Medical), which have implemented software algorithms for automated detection of CFAEs. Both algorithms are based on time-domain analysis. However, the results of these algorithms depend on initial settings of specific parameters and for this reason vary with different operators [7]. Up to now there is only one algorithm published by Kremen and Lhotska [5] for the purpose of searching for individual signal complexes in CFAEs. Based on wavelet transformation their algorithm uses the affinity of the signal to a wavelet in a certain frequency band for classification.

In this paper we present a new approach to segment the intracardiac electrograms into sections of signal complexes related to electrical activity and into those where an isoelectric line is dominant. Our method is based on the idea of the Pan-Tompkins' QRS-detection algorithm [8]. Instead of using differentiation operator we use Teager's energy operator [9] for signal feature extraction. By observing the signal energy, not only the frequency but also the amplitude is taken into consideration. Thus, our algorithm shows a robust performance even if the baseline is highly erratic.

\section{MATERIALS}

Intracardiac electrograms of patients with paroxysmal or persistent AFib were recorded during endocardial mapping at roof, septum, anterior and posterior wall of the left atrium. A Lasso ${ }^{\circledR}$ catheter (Biosense Webster) with 10 electrodes is used, which provides nine leads of bipolar intracardiac electrograms. The potential difference between two adjacent electrodes is given at each lead. The data is recorded by Ensite NavX system (St. Jude Medical) with a sample frequency of $1.2 \mathrm{kHz}$. Each recording has a length of 5 or 6 seconds. The data is prefiltered by the measurement system. 


\section{Methods}

\section{A. Teager's energy operator}

Working in the field of nonlinear speech processing, Teager proposed a simple Non-Linear Energy Operator (NLEO) [9] for time-discrete signals given as follows

$$
E_{n}=E[x(n)]=x_{n}^{2}-x_{n+1} x_{n-1} .
$$

Kaiser showed in his analysis, that NLEO can detect frequency and amplitude of the examined signals [9]. For a harmonic signal NLEO yields

$$
E_{n}=E[A \cos (\Omega n+\Phi)]=A^{2} \sin ^{2} \Omega,
$$

where $\Omega$ is the digital frequency in radians/sample and is given by $\Omega=2 \pi f / f_{s}$. $f$ is the analog frequency and $f_{s}$ the sample frequency. $A$ is the amplitude and $\Phi$ the arbitrary initial phase. For small $\Omega$ we obtain

$$
E_{n}=E[A \cos (\Omega n+\Phi)]=A^{2} \Omega^{2} .
$$

With $\Omega<\pi / 4$ or $f / f_{s}<1 / 8$ the relative error in the last approximation is always less than $11 \%$. Now let us consider $x(t)=A \cos (\omega t+\Phi)$ the time-continuous counterpart of $x(n)$. $x(t)$ can be interpreted as the solution of the well-known second order differential equation

$$
\ddot{x}+\frac{k}{m} x=0
$$

which describes the motion of a mass $m$ suspended by a spring with force constant $k$. The energy of the mass-spring system substituted with $x(t)$ is

$$
E=\frac{1}{2} k x^{2}+\frac{1}{2} m \dot{x}^{2}=\frac{1}{2} m \omega^{2} A^{2}
$$

and thus, $E \propto A^{2} \omega^{2}$. Comparing this result with equation (3), we see that the output of NLEO can be considered as an indication of the energy of the signal $x(n)$.

\section{B. Segmentation of intracardiac electrograms}

During endocardial mapping electrophysiological activity is marked by changes in amplitude and frequency in intracardiac electrograms. It is desired to locate these "active sections" and separate them from sections where isoelectric line is expected - called "inactive sections". As Teager's NLEO provides a quantity which is proportional to the (squared) amplitude and the (squared) frequency of the signal, we use the relative value of this quantity as the extracted feature for automated classification. Table 1 shows the relation between the steps in Pan-Tompkins' algorithm and those in the approach presented in this paper.

Table 1: Comparison between Pan-Tompkins' method and our approach

\begin{tabular}{lll}
\hline & Pan-Tompkins' algorithm & New approach in this paper \\
\hline 1 & Preprocessing & Preprocessing \\
2 & Differentiation \& Squaring & Evaluation with NLEO \\
3 & Moving-Window Integration & Gaussian Lowpass Filtering \\
4 & Thresholding & Thresholding \\
5 & Postprocessing & Postprocessing \\
\hline
\end{tabular}

Preprocessing In the first step, baseline wander is eliminated by a $\mathrm{DWT}^{1}$-based approach [10] where frequencies in range $0-1.17 \mathrm{~Hz}$ are removed. Moreover, high frequency noise is eliminated from the atrial electrograms by a generalized equiripple lowpass filter. For the later application of NLEO, the filter stopband corner frequency $f_{\text {stop }}$ (with an attenuation of $-32 \mathrm{~dB}$ ) is chosen to be one-eighth of the sample frequency, which equals to $150 \mathrm{~Hz}$. Since frequencies greater than $150 \mathrm{~Hz}$ are beyond the physiological frequency range, this restriction does not lead to information loss. The signal after preprocessing step is shown in Fig. 1(a).

Evaluation with NLEO The output of NLEO highlights sections with high frequency and high amplitude. In Fig. 1(b) we note that the relative difference between NLEO's output of sections where the signal is "active" and where the signal is "inactive", is significant.

Gaussian Lowpass Filtering In order to take the width of an individual peak into account, the NLEO output is filtered with a lowpass. For this purpose, a gaussian filter is chosen because both - its impulse response and frequency response are smooth in the range of interest, Fig. 2.

According to Pan \& Tompkins, the effective width of the filter impulse response should be in the order of the average peak width. In intracardiac electrograms peaks are usually 10-20 ms wide. The effective width of the filter impulse response is set to 17 samples, Fig. 2(a), which, in case of a sample frequency of $1200 \mathrm{~Hz}$, corresponds to $14 \mathrm{~ms}$.

In spectral analysis of intracardiac electrograms, the frequency range of interest is $0-20 \mathrm{~Hz}$ [11]. Within this range, the magnitude spectrum of the electrograms often shows a single dominant peak with its harmonics. For that reason, the cutoff frequency $f_{c}$ is set to $24 \mathrm{~Hz}$, Fig. 2(b). The lowpass filtered NLEO's output is shown in Fig. 1(c).

\footnotetext{
${ }^{1}$ Discrete Wavelet Transformation
} 


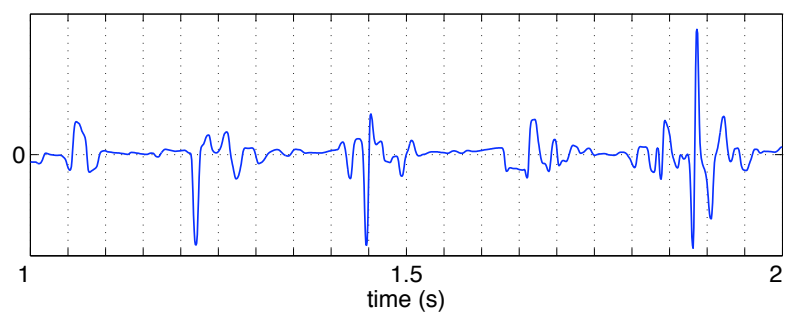

(a)

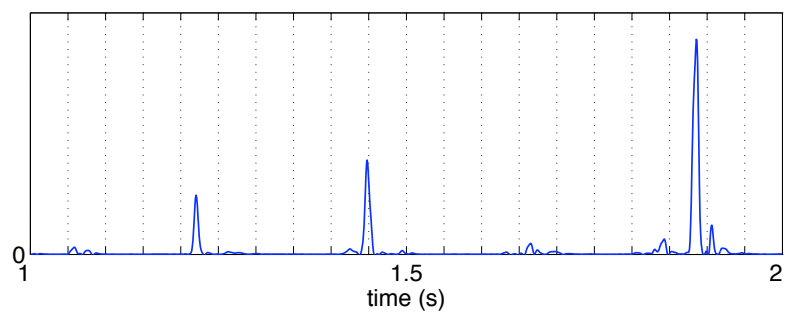

(b)

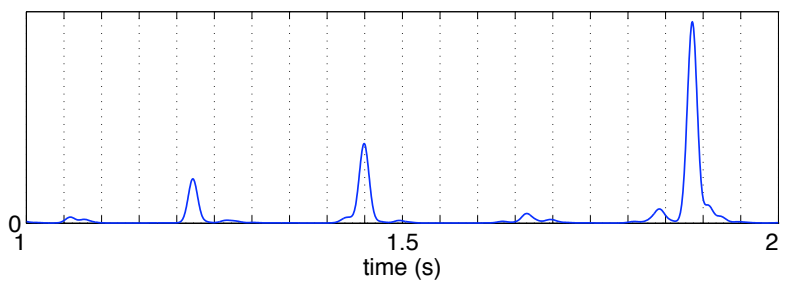

(c)

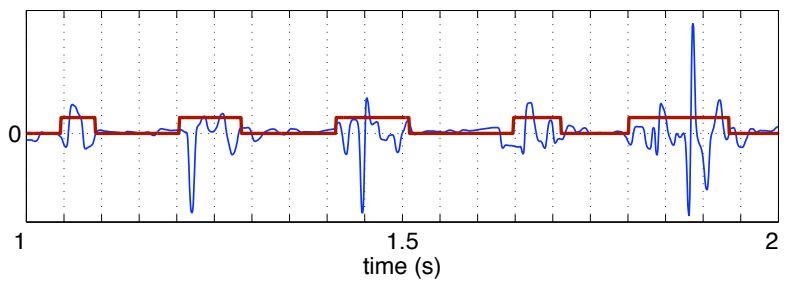

(d)

Fig. 1: Visualization of the segmentation algorithm in its steps. (a) Signal after preprocessing. (b) NLEO's output: the signal "energy". (c) Filtered NLEO's output. (d) Result after thresholding.

Thresholding To adapt to changing characteristics of the signal, thresholds are adjusted periodically after $\Delta t$. Over a one-second time window $w_{i}$ the threshold $\theta_{i}$ is calculated using a factor $k$ of the standard deviation of the filtered energy within $w_{i}$. The time shift $\Delta t$ is set to $50 \mathrm{~ms}$. Thus, every data point has 20 thresholds $\theta_{i}(n)$ of 20 shifted windows $w_{i}$, except for those lying in the first and the last second of the data set. For each data point, we choose the threshold $\theta(n)=\min \left(\theta_{i}(n)\right), i=1, \ldots, 20$.

Finally, the factor $k$ is the only arbitrary parameter, which is used to weight the standard deviation of the filtered energy. According to experts' validation, $k$ is set to 0.1 .

By comparing the filtered energy in Fig. 1(c) with the adaptive thresholds $\theta(n)$, sections with high energy (active section) are located and separated from those with low energy (inactive section). The result is shown in Fig. 1(d).

Postprocessing In the last step the width of inactive and active sections are revised. All inactive sections, which are shorter than a refractory period of $30 \mathrm{~ms}$, are removed and set to be active. In those cases neighboring active sections are merged.

The respective procedure is applied to the active sections: an active section should not be shorter than $10 \mathrm{~ms}$.

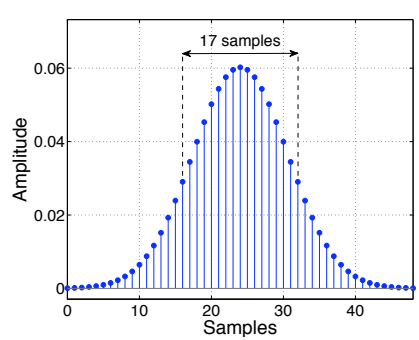

(a) Impulse response

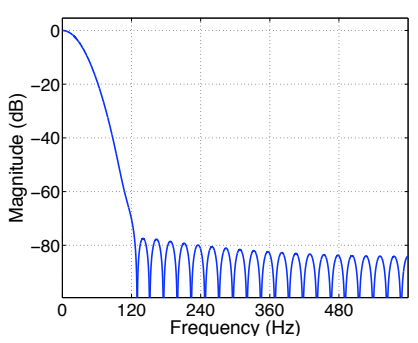

(b) Frequency response
Fig. 2: Gaussian lowpass filter

\section{Discussions \& Results}

A new method for automated location and segmentation of active segments in intracardiac electrograms is proposed. This method has been applied on recordings from different positions in the left atrium of five patients with paroxysmal or persistant AFib. The results were validated by experts.

The presented method uses the signal energy as an extracted feature for classification. Since both signal frequency and signal amplitude are taken into consideration, results indicate high performance and stability. Unlike methods based on the analysis of the signal frequency, perturbation of the baseline does not affect the result of our algorithm.

With adaptive thresholding, changes in signal characteristic can be detected and evaluated accordingly. Fig. 3(a) shows that active sections with high amplitudes as well as active sections with low amplitudes are located accurately with the presented method.

During endocardial mapping, CFAEs are in the focus of interest since they are associated with AFib substrate. In atrial 
electrograms, sections with a prolonged activation complex are key indices for CFAEs, Fig. 3(b). By introducing a refractory period, fractionated sections can be identified in their true length. This is achieved in the postprocessing step, where adjacent active sections with an intersegment smaller than the refractory period are merged. Therefore active sections in the segmented signal are able to represent the local electrophysiological activities.

This method has also been tested on intracardiac electrograms during atrial flutter and sinus rhythm. The results are plausible since in both cases the baseline is smoother than during AFib. With accordingly adjusted parameters (average peak width, length of the time-window to calculate thresholds and refractory period), this method works as well on surface ECGs for detection of QRS-complexes.

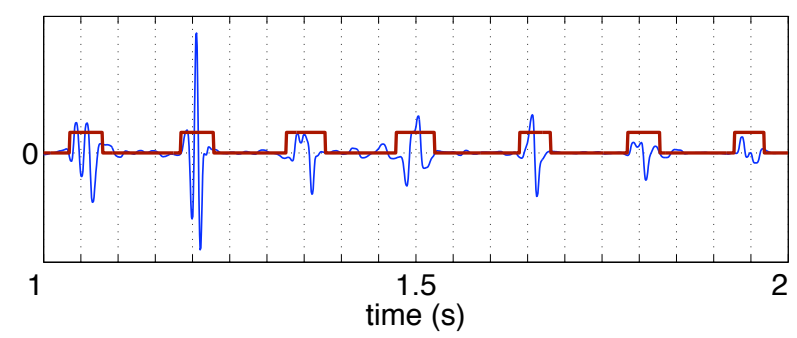

(a)

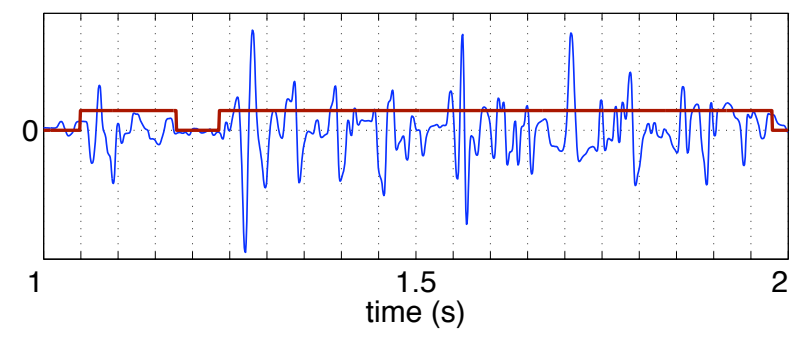

(b)

Fig. 3: Examples of segmented intracardiac electrograms. On a signal with changing amplitudes in (a) and with continuous electrical activity in (b).

\section{CONCLUSION}

With this approach we reach our goal of providing a reliable algorithm for location and segmentation of signal complexes in intracardiac electrograms. Objective assessment of the local electrical activity in intracardiac electrograms can be achieved with the presented algorithm.
Based on the segmented data, further investigation can be performed to extract more features from the active signal complexes, such as fractionation evaluation for automated diagnosis of AFib. With its low computational complexity, this method is capable for real-time application and can be an operator-independent guide for electrophysiologists during catheter ablation.

Because of its nature, this algorithm works not only with intracardiac electrograms during atrial fibrillation, atrial flutter or sinus rhythm, but also with surface ECGs. So far this is a theoretical approach and needs further investigations to warrant its clinical value.

\section{REFERENCES}

1. Allessie MA, Konings K., Kirchhof C., Wijffels M. (1996) Electrophysiologic mechanisms of perpetuation of atrial fibrillation. The American Journal of Cardiology 77:10-23.

2. Haissaguerre M., Jais P., Shah D.C., et al. (1998) Spontaneous initiation of atrial fibrillation by ectopic beats originating in the pulmonary veins. New England Journal of Medicine 339:659-666.

3. Nademanee K., McKenzie J., Kosar E., et al. (2004) A new approach for catheter ablation of atrial fibrillation: mapping of the electrophysiologic substrate. Journal of the American College of Cardiology 43:20442053.

4. Haissaguerre M., Wright M., Hocini M., Jais P. (2008) The substrate maintaining persistent atrial fibrillation. Circulation: Arrhythmia and Electrophysiology 1:2-5.

5. Kremen V., Lhotsha L. (2007) Novel approach to search for individual signal complexes in complex fractionated atrial electrograms using wavelet transform. 6th International Special Topic Conference on ITAB, :83-86.

6. Ng J., Goldberger J.J. (2007) Understanding and interpreting dominant frequency analysis of AF electrograms. Journal of Cardiovascular Electrophysiology 18:680-685.

7. Aizer A., Holmes D.S., Garlitski A.C., et al. (2008) Standardization and validation of an automated algorithm to identify fractionation as a guide for atrial fibrillation ablation. Heart Rhythm 5:1132-1141.

8. Pan J., Tompkins W.J. (1985) A Real-Time QRS Detection Algorithm. IEEE Transactions on Biomedical Engineering 32:230-236.

9. Kaiser J.F. (1990) On a simple algorithm to calculate the 'energy' of a signal. International Conference on Acoustics, Speech, and Signal Processing 1:381-384.

10. Khawaja A. (2007) Automatic ECG analysis using principal component analysis and wavelet transformation. Universitätsverlag, Karlsruhe.

11. Everett T.H., Kok Lai-Chow, Vaughn R.H., Moorman R., Haines D.E. (2001) Frequency domain algorithm for quantifying atrial fibrillation organization to increase defibrillation efficacy. IEEE Transactions on Biomedical Engineering 48:969-978.

$\begin{array}{ll}\text { Corresponding Author: } & \begin{array}{l}\text { Christopher Schilling } \\ \text { Institute of Biomedical Engineering, } \\ \text { Unstitute: }\end{array} \\ \text { Universität Karlsruhe (TH) } \\ \text { Street: } & \text { Kaiserstrasse 12 } \\ \text { City: } & \text { 76131 Karlsruhe } \\ \text { Country: } & \text { Germany } \\ \text { Email: } & \text { Christopher.Schilling@ibt.uni-karlsruhe.de }\end{array}$

